\title{
IS GUM ARABIC A GOOD EMULSIFIER DUE TO CH... $\pi$ INTERACTIONS? HOW UREA EFFECTIVELY DESTABILIZES THE HYDROPHOBIC CH.... INTERACTIONS IN THE PROTEINS OF GUM ARABIC THAN AMIDES AND GUHCI?
}

\author{
Sowmiya Sethuraman†, Kumaran Rajendran*†. \\ † D.G. Vaishnav College, Autonomous (affiliated to the University of the Madras (Chennai)) Department of \\ Chemistry, 833, Periyar EVR Salai, Arumbakkam, Chennai-600 106, Tamil Nadu, India
}

Sample details: Merck Chemicals provided the following details.

CAS Number: 9000-01-5

EC Number: 232-519-5

Species: Acacia Senegal

Grade Value: Ph Eur, BD. Acacia, Arabic Gum

HS Code: 13012010

Density: $1.4 \mathrm{~g} / \mathrm{cm}^{3}$

$\mathrm{pH}: 5$

\section{Steady-state fluorescence measurements:}

The excitation and emission slits were set at $5 \mathrm{~nm}$ for all the 3D measurements. The excitation wavelength range were 250 to $500 \mathrm{~nm}$ and the emission wavelength range were 280 to $550 \mathrm{~nm}$. The emission maximum corresponding to each excitation wavelength was recorded and a plot was generated using Origin 8.0 Software.

Time correlated single-photon counting (TCSPC) technique: TCSPC is a digital technique, counts the photons, which are time correlated with the excitation pulse. The heart of the method is a time-to-amplitude converter (TAC). The fluorescence photons are collected at the magic angle (54.7) to avoid the distortions due to the rotational polarization. The measured decay is the convolution of the true fluorescence decay, excitation function and the instrument response function which results in the fluorescence kinetics parameters like lifetime $(\tau)$ and relative amplitude(A). The data analysis was carried out by the software provided by IBH (DAS-6) which is based on reconvolution technique using iterative non-linear least square methods. The reconvolution is preceded by the series of iterations until a chi-square is reduced. The quality of fit is normally identified by the reduced $\chi 2$, weighed residual and auto correlation function of the residuals.
Figure S1. Three-dimensional (3D) emission contour spectrum of GA $\left[2.86 \times 10^{-6} \mathrm{M}\right]$ in the presence of $\mathrm{GuHCl}$ $\left[1.0 \times 10^{-1} \mathrm{M}\right]$.

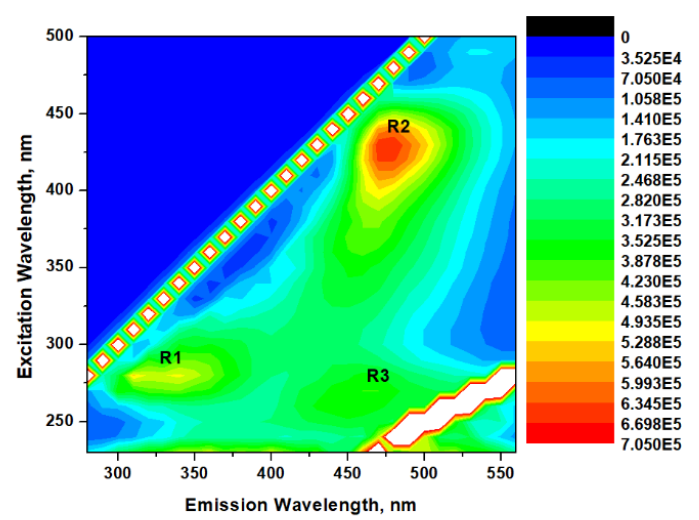

Figure S2. Three-dimensional (3D) emission contour spectrum of GA $\left[2.86 \times 10^{-6} \mathrm{M}\right]$ in the presence of DMU $\left[1.0 \times 10^{-1} \mathrm{M}\right]$.

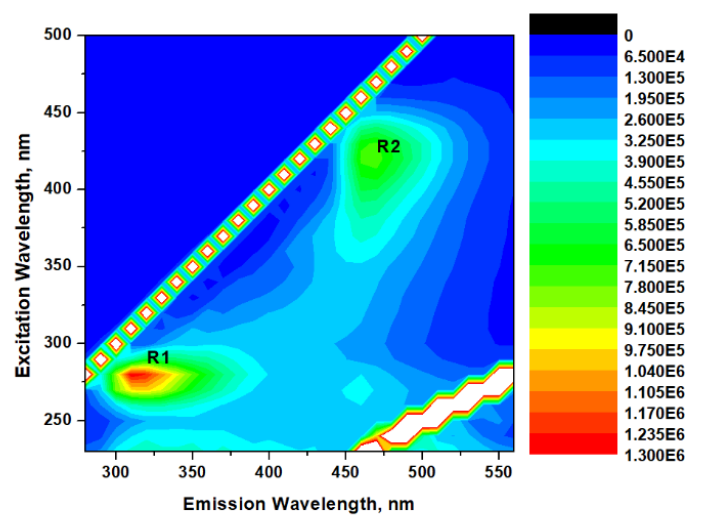


Figure S3. Three-dimensional (3D) emission contour spectrum of GA $\left[2.86 \times 10^{-6} \mathrm{M}\right]$ in the presence of DMU $[3.0 \mathrm{M}]$.
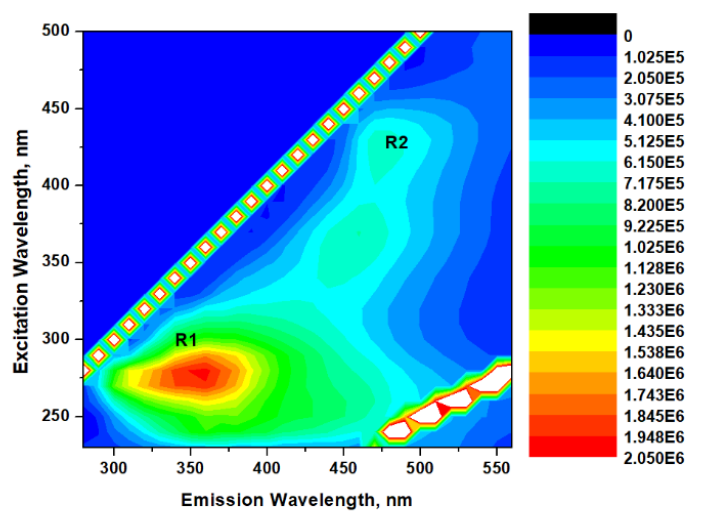

Figure S4. Three-dimensional (3D) emission contour spectrum of GA $\left[2.86 \times 10^{-6} \mathrm{M}\right]$ in the presence of DMU $[5.0 \mathrm{M}]$.

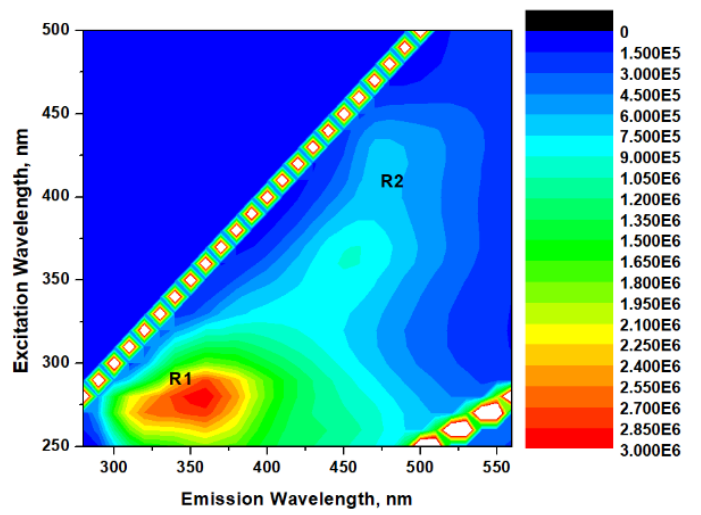

Figure S5. Three-dimensional (3D) emission contour spectrum of GA $\left[2.86 \times 10^{-6} \mathrm{M}\right]$ in the presence of TMU [1.0 M].

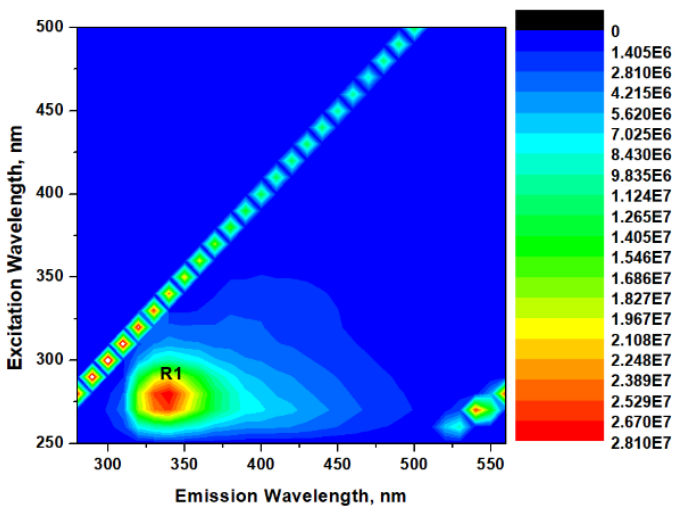

Figure S6. Three-dimensional (3D) emission contour spectrum of GA $\left[2.86 \times 10^{-6} \mathrm{M}\right]$ in the presence of TMU [3.0 M].

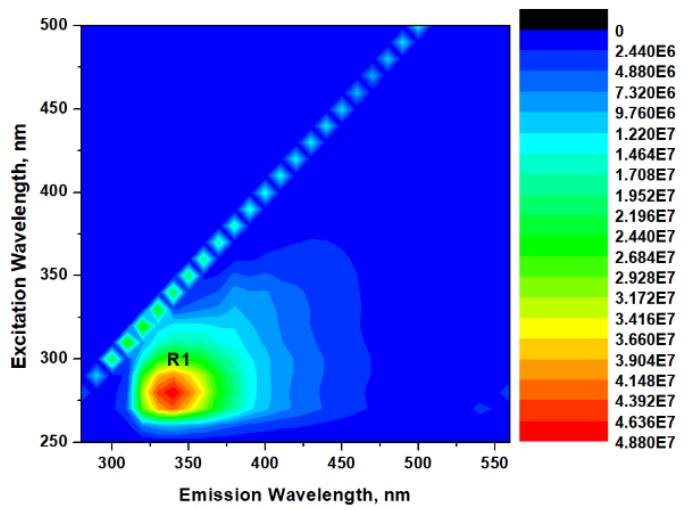

Figure S7. Fluorescence decay of GA in the absence and presence of GuHCl.

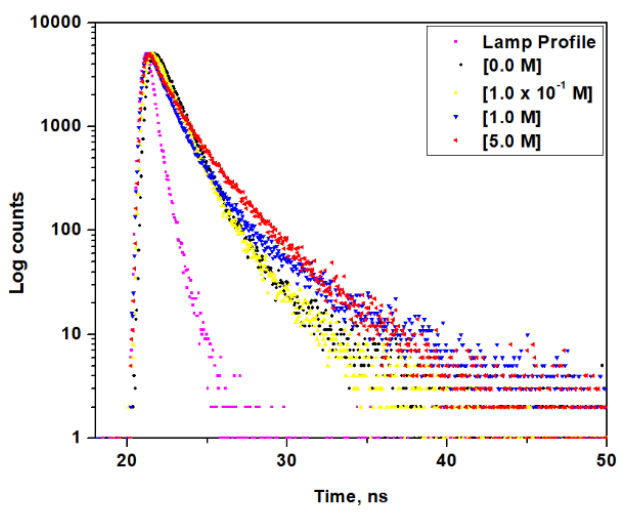

Figure S8. Fluorescence decay of GA in the absence and presence of AA.

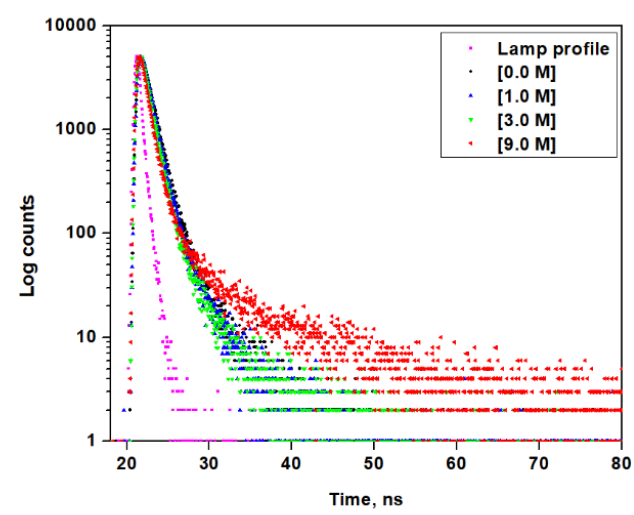


Table S1. Fluorescence lifetime decay analysis of GA in the absence and presence of Urea.

\begin{tabular}{|c|c|c|c|c|c|c|c|c|c|c|c|}
\hline \multirow[t]{2}{*}{ [Urea] M } & \multicolumn{6}{|c|}{ Fluorescence Lifetime (ns) } & \multicolumn{3}{|c|}{ Relative Amplitude \% } & \multirow[t]{2}{*}{$\chi^{2}$} & \multirow{2}{*}{$\begin{array}{c}\text { Average } \\
\text { Lifetime } \\
\tau \text { (ns) }\end{array}$} \\
\hline & $\tau 1$ & \pm & $\tau 2$ & \pm & $\tau 3$ & \pm & $\mathrm{A} 1$ & $\mathrm{~A} 2$ & A3 & & \\
\hline $\mathbf{0}$ & 0.11 & 0.13 & 0.97 & 0.06 & 2.88 & 0.3 & 19 & 70 & 11 & 1.08 & 1.0167 \\
\hline 0.1 & 0.16 & 0.01 & 1.46 & 0.09 & 3.63 & 0.07 & 40 & 30 & 30 & 0.95 & 1.591 \\
\hline 1 & 0.11 & 0.02 & 1.47 & 0.08 & 3.81 & 0.14 & 44 & 35 & 21 & 1.15 & 1.363 \\
\hline 5 & 0.11 & 0.02 & 1.26 & 0.07 & 3.23 & 0.06 & 38 & 31 & 31 & 1.26 & 1.4337 \\
\hline
\end{tabular}

Table S2. Fluorescence lifetime decay analysis of GA in the absence and presence of GuHCl.

\begin{tabular}{|c|c|c|c|c|c|c|c|c|c|c|c|}
\hline \multirow[t]{2}{*}{ [GudHCl] M } & \multicolumn{6}{|c|}{ Fluorescence Lifetime (ns) } & \multicolumn{3}{|c|}{ Relative Amplitude \% } & \multirow[t]{2}{*}{$\chi^{2}$} & \multirow{2}{*}{$\begin{array}{l}\text { Average } \\
\text { Lifetime } \\
\tau \text { (ns) }\end{array}$} \\
\hline & $\tau 1$ & \pm & $\tau 2$ & \pm & $\tau 3$ & \pm & A1 & $\mathrm{A} 2$ & A3 & & \\
\hline $\mathbf{0}$ & 0.11 & 0.13 & 0.97 & 0.06 & 2.88 & 0.3 & 19 & 70 & 11 & 1.08 & 1.0167 \\
\hline 0.1 & 0.35 & 0.15 & 0.96 & 0.04 & 2.88 & 0.12 & 14 & 74 & 13 & 1.04 & 1.1338 \\
\hline 1 & 0.46 & 0.05 & 1.36 & 0.05 & 4.79 & 0.19 & 37 & 50 & 13 & 0.89 & 1.4729 \\
\hline 5 & 0.1 & 0.005 & 1.17 & 0.05 & 2.95 & 0.05 & 35 & 34 & 31 & 1.07 & 1.3473 \\
\hline
\end{tabular}

Table S3. Fluorescence lifetime decay analysis of GA in the absence and presence of DMU.

\begin{tabular}{|c|c|c|c|c|c|c|c|c|c|c|c|}
\hline \multirow[t]{2}{*}{ [DMU] M } & \multicolumn{6}{|c|}{ Fluorescence Lifetime (ns) } & \multicolumn{3}{|c|}{ Relative Amplitude } & \multirow[t]{2}{*}{$\chi^{2}$} & \multirow{2}{*}{$\begin{array}{l}\text { Average } \\
\text { Lifetime } \\
\tau \text { (ns) }\end{array}$} \\
\hline & $\tau 1$ & \pm & $\tau 2$ & \pm & $\tau 3$ & \pm & $\mathrm{A} 1$ & $\mathrm{~A} 2$ & A3 & & \\
\hline $\mathbf{0}$ & 0.11 & 0.13 & 0.97 & 0.06 & 2.88 & 0.3 & 19 & 70 & 11 & 1.08 & 1.0167 \\
\hline 1.5 & 0.08 & 0.006 & 1.06 & 0.02 & 3.59 & 0.2 & 24 & 67 & 10 & 1.1 & 1.0884 \\
\hline 2.5 & 0.41 & 0.16 & 1.17 & 0.03 & 3.76 & 0.14 & 10 & 77 & 12 & 1.17 & 1.3931 \\
\hline 4.5 & 1.13 & 0.1 & 2.17 & 0.18 & 7.1 & 0.42 & 62 & 28 & 10 & 1.06 & 2.0182 \\
\hline
\end{tabular}

Table S4. Fluorescence lifetime decay analysis of GA in the absence and presence of TMU.

\begin{tabular}{|c|c|c|c|c|c|c|c|c|c|c|c|}
\hline \multirow[t]{2}{*}{ [TMU] M } & \multicolumn{6}{|c|}{ Fluorescence Lifetime (ns) } & \multicolumn{3}{|c|}{ Relative Amplitude \% } & \multirow[t]{2}{*}{$\chi^{2}$} & \multirow{2}{*}{$\begin{array}{l}\text { Average } \\
\text { Lifetime } \\
\tau \text { (ns) }\end{array}$} \\
\hline & $\tau 1$ & \pm & $\tau 2$ & \pm & $\tau 3$ & \pm & A1 & A2 & A3 & & \\
\hline $\mathbf{0}$ & 0.11 & 0.13 & 0.97 & 0.06 & 2.88 & 0.3 & 19 & 70 & 11 & 1.08 & 1.0167 \\
\hline 1 & 26.43 & 0.66 & 1.11 & 0.06 & 4.51 & 0.56 & 76 & 10 & 14 & 1.02 & 20.6932 \\
\hline 2 & 36.58 & 0.39 & 2.11 & 0.22 & 7.11 & 0.25 & 77 & 6 & 17 & 0.96 & 29.5019 \\
\hline 3 & 42.63 & 0.71 & 2.85 & 0.2 & 8.39 & 0.33 & 73 & 6 & 20 & 0.96 & 32.9689 \\
\hline 4 & 39.89 & 0.6 & 2.49 & 0.36 & 7.36 & 0.23 & 73 & 5 & 22 & 1.03 & 30.8634 \\
\hline
\end{tabular}


Table S5. Fluorescence lifetime decay analysis of GA in the absence and presence of AA.

\begin{tabular}{|c|c|c|c|c|c|c|c|c|c|c|c|}
\hline \multirow[t]{2}{*}{ [AA] M } & \multicolumn{6}{|c|}{ Fluorescence Lifetime (ns) } & \multicolumn{3}{|c|}{ Relative Amplitude } & \multirow[t]{2}{*}{$\chi^{2}$} & \multirow{2}{*}{$\begin{array}{l}\text { Average } \\
\text { Lifetime } \\
\tau \text { (ns) }\end{array}$} \\
\hline & $\tau 1$ & \pm & $\tau 2$ & \pm & $\tau 3$ & \pm & A1 & A2 & A3 & & \\
\hline 0.0 & 0.11 & 0.13 & 0.97 & 0.06 & 2.88 & 0.3 & 19 & 70 & 11 & 1.08 & 1.0167 \\
\hline 1.0 & 0.33 & 0.09 & 0.88 & 0.03 & 2.88 & 0.1 & 23 & 68 & 9 & 1.07 & 0.9335 \\
\hline 3.0 & 0.6 & 0.2 & 1.13 & 0.11 & 4.79 & 1.08 & 43 & 49 & 8 & 1.13 & 1.1949 \\
\hline 9.0 & 0.52 & 0.03 & 1.46 & 0.09 & 7.33 & 0.46 & 70 & 21 & 8 & 1.19 & 1.257 \\
\hline
\end{tabular}

